\title{
High Sensitive Method Development and validation of Imotriptan in Human Plasma by UPLC Tandem Mass Spectrometry
}

\author{
G. Shiva Kumar ${ }^{1}$, Dr. Jaya Dwivedi ${ }^{2}$ \\ ${ }^{1}$ Application Department, Waters India, Benguluru, India. \\ ${ }^{2}$ Department of Chemistry, Banasthali Vidyapeeth, Jaipur, Rajasthan.
}

\begin{abstract}
A highly sensitive ultra-high performance liquid chromatography tandem mass spectrometry (UPLC-MS/MS) method was developed for the quantification of Almotriptan in human plasma.To the best of our knowledge; this is the sensitive method for the quantification of Almotriptan in human plasma. Matrix effects were strongly reduced and selectivity increased by using an efficient chromatographic separation on a sub-2 $2 \mu \mathrm{m}$ column (Acquity UPLC HSS T3 $1.8 \mu \mathrm{m}, 2.1 \times 50 \mathrm{~mm}$ ) in 3 min with a gradient of ammonium formate $5 \mathrm{mM}$ and acetonitrile as mobile phase at a flow rate of $0.4 \mathrm{ml} / \mathrm{min}$. Detection was made using a tandem quadrupole mass spectrometer operating in positive electrospray ionization mode, using multiple reaction monitoring. The procedure was fully validated according to the latest Food and Drug Administration guidelines. Very good results were obtained by using Naratriptan as internal standard, to compensate for the variability due to the extraction and ionization steps. The method was very sensitive with lower limits of quantification of $0.1 \mathrm{ng} / \mathrm{ml}$ for Almotriptan. The upper limit of quantification was $250 \mathrm{ng} / \mathrm{ml}$. Accuracy $(94.0$ $105.7 \%)$, Precision (1.0-6.7\%), and internal standard-normalized matrix effects (95-102\%) were in accordance with international recommendations. The method can be used for pharmacokinetic studies.
\end{abstract}

Keywords: Almotriptan, Human plasma, Multiple reaction monitoring, Quantification, Solid Phase Extraction, UPLC-MS/MS

\section{Introduction:}

Almotriptan is prescribed to treat the acute headache phase of migraine attacks with or without aura [1]. Almotriptan is the only oral triptan approved in the USA for the treatment of migraine in adolescent from 12 to 17 years of age. Almotriptan has a high and specific affinity for serotonin $5-\mathrm{HT}_{1 \mathrm{~B} / 1 \mathrm{D}}$ receptors. Binding of the drug to the receptor leads to vasoconstriction of the cranial blood vessels and thus affects the redistribution of cranial blood flow. Almotriptan significantly increases cerebral blood flow and reduces blood flow through extracerebral cranial vessels. Even though it affects cranial blood vessels a single dose of almotriptan $(12.5 \mathrm{mg})$ has no clinically significant effect on blood pressure or heart rate in both young and elderly healthy volunteers. However larger doses seem to slightly increase blood pressure but not beyond clinical relevance [2]. Almotriptan has a linear pharmacokinetic up to $200 \mathrm{mg}$ dose. Its half life is 3 hours and nearly $70 \%$ bioavailability. $\mathrm{C}_{\max }$ is observed $1.5-4$ hours after oral administration and approximately $50 \%$ of the drug is excreted unchanged in the urine. Metabolism is mediated through MAO-A and CYP3A4 and CYP2D6 oxidation. Almotriptan clearance is moderately reduced in the elderly but does not require dose adjustment. Sex does not alter the pharmacokinetics of the drug. However, patients with moderate-to-severe renal dysfunction should limit their total daily dose to $12.5 \mathrm{mg}$ [3]. To our knowledge, several methods for the determination of Almotriptan in biological matrixes [4], pharmaceutical formulations by LC-MS/MS, HPLC HPTLC, fluorimetric and calorimetry have been reported. The purpose of this study was to develop and validate a novel sensitive LC-MS/MS method to quantify Almotriptan in human plasma.

\subsection{Chemicals and reagents:}

\section{Experimental:}

Almotriptan maleate and Naratriptan Figure 1 was obtained from Sigma Aldrich, India. All other chemicals (Ammonium formate, formic acid, acetonitrile, and methanol) and solvents were purchased from Fluka Mumbai. Oasis HLB $1 \mathrm{~cm}^{3}(30 \mathrm{mg})$ extraction cartridges were purchased from Waters Corporation (Benguluru, India). Human plasma samples used for method development and validation were obtained from Vidhi Laboratory (Ahmedabad, India) and were stored at $-20{ }^{\circ} \mathrm{C}$ until use. Six different batches were tested for the absence of studied analytes prior to their use.

\subsection{Stock and Working solutions:}

Stock solutions of each analyte at $1 \mathrm{mg} / \mathrm{ml}$ in methanol were stored at $2-8^{\circ} \mathrm{c}$. Working solutions containing analyte at different concentrations were obtained by dilution of the stock solutions with water 
methanol at $(1: 1)$ ratio. Blank plasmas were spiked with the appropriate working solution to prepare independently the calibration and the validation standard samples at the appropriate concentrations. Stock solutions of Naratriptan at $0.1 \mathrm{mg} / \mathrm{ml}$ in methanol were stored at $2-8{ }^{\circ} \mathrm{C}$. A working solution containing the Naratriptan at $10 \mathrm{ng} / \mathrm{ml}$ in water methanol at $(1: 1)$ ratio was prepared and stored at $2-8{ }^{\circ} \mathrm{C}$.

\subsection{Equipment:}

The liquid chromatography system consisted of a Waters Acquity UPLC instrument equipped with a binary pump and a 96-vial autosampler (Waters, Milford, USA). Chromatographic sepearation was performed on an Acquity UPLC HSS T3 $1.8 \mu \mathrm{m}, 2.1 \times 50 \mathrm{~mm}$ column with the corresponding guard column (Waters). The chromatographic system was coupled to a tandem quadrupole mass spectrometer (Xevo TQMS, Waters) operating in positive electrospray ionization mode (ESI+). Instrument control and data acquisition were performed with MassLynx software V4.1 (Waters).

\subsection{UPLC-MSMS Conditions:}

Chromatographic separation was performed at a flow rate of $0.4 \mathrm{ml} / \mathrm{min}$. A gradient of mobile phase composed of ammonium formate buffer $5 \mathrm{mM}$ (solution A) and ACN (solution B) was used with the following elution program (B\%): $10 \%$ at $0.0 \mathrm{~min}$, held for $0.5 \mathrm{~min}, 90 \%$ at $1.75 \mathrm{~min}$, and held for $0.5 \mathrm{~min}$ then with in 0.25 min back to initial conditions. The system was then placed in the initial mobile phase condition during the preparation of the next injection $(0.5 \mathrm{~min})$. The injection volume was set at $5 \mu \mathrm{l}$ in full loop mode. The injection system was washed between each run with $300 \mu \mathrm{l}$ of strong wash [ACN-methanol-isopropanol-water with $0.1 \%$ Formic acid $(25: 25: 25: 25, \mathrm{v} / \mathrm{v} / \mathrm{v} / \mathrm{v})]$, followed by $900 \mu \mathrm{l}$ of weak wash [ACN-water $(10: 90, \mathrm{v} / \mathrm{v})]$. Column temperature was set at $40{ }^{\circ} \mathrm{C}$ and auto sampler was kept at $10{ }^{\circ} \mathrm{C}$. Detection was achieved using multiple reaction monitoring (MRM) mode. Nitrogen was used as desolvation gas at a temperature of $500{ }^{\circ} \mathrm{C}$ and a flow rate of $1000 \mathrm{l} / \mathrm{hr}$, and as cone gas at a flow rate of $50 \mathrm{l} / \mathrm{hr}$. Argon was used as collision gas at a flow rate of 0.17 $\mathrm{ml} / \mathrm{min}$. Capillary voltage was set at $3 \mathrm{kV}$ and source temperature at $150{ }^{\circ} \mathrm{C}$. Using two functions (time segment), dwell time was $200 \mathrm{~ms}$ for each ion. MRM transition states, cone voltages and collision energies are shown in Table 1 for each compound.

\subsection{Sample Preparation:}

Sample preparation was performed using oasis HLB catridges. All previously frozen plasma samples were thawed at room temperature and vortexed for proper mixing. $10 \mu 1$ of internal standard Naratriptan at concentration $10 \mathrm{ng} / \mathrm{ml}$ was added to $100 \mu \mathrm{l}$ aliquot of human plasma. The tubes were properly vortexed for mixing and then plasma samples were pretreated with water and vortexed .HLB cartridges were conditioned with methanol and water. After loading the sample cartridges were washed with water and then with 5\% methanol eluted with methanol. Dried the eluent at $40^{\circ} \mathrm{c}$ and reconstituted with $5 \mathrm{mM}$ ammonium Formate and Acetonitrile (7:3). $5 \mu$ l of the sample was injected onto the column.

\subsection{Method Validation:}

The method was fully validated based on the latest US Food and Drug Administration (FDA) [5] and European Medicines Agency (EMA) guidelines for bioanalytical method validation [6].

\subsubsection{Selectivity and carry-over:}

The method selectivity toward matrix was assessed by analyzing blank plasma samples from 6 different batches for the presence of interfering peaks at analytes retention time. Carry-over effects were assessed by injecting reconstitution solution [buffer/ACN (30:70, v/v)] after the highest calibration sample.

\subsubsection{Matrix effects, extraction recovery and process efficiency:}

Matrix effects were qualitatively evaluated by post-column infusion. Analytes and their IS were continuously infused at $10 \mu \mathrm{l} / \mathrm{min}$ into the detector during chromatographic analysis of a mobile phase, an extracted water and 6 different blank plasma extracts. Almotriptan and its IS were infused at a concentration of $10 \mathrm{ng} / \mathrm{ml}$, which corresponded to a signal response of approximately 3 times the lower limit of quantification (LLOQ). Alterations of the signal at the retention times of the analytes were considered as matrix effects as it could modify analytes' quantification. Samples at low $(0.1 \mathrm{ng} / \mathrm{ml}$, close to LLOQ) and high $(250 \mathrm{ng} / \mathrm{ml})$ concentrations were used. IS concentrations were set at fixed concentration $(10 \mathrm{ng} / \mathrm{ml})$ for all samples. Three different sets of samples were prepared: water samples spiked after extraction in triplicates (A) and 6 different blank plasma samples spiked after extraction (B) or before extraction (C) in duplicates. Matrix effect (ME) was evaluated as the ratio of peak areas from post-extraction spiked plasmas and post-extraction spiked water samples $(\mathrm{ME}=\mathrm{B} / \mathrm{A})$. Extraction recovery $(\mathrm{RE})$ was estimated as the ratio of peak areas from pre- and postextraction spiked plasmas $(\mathrm{RE}=\mathrm{C} / \mathrm{B})$. Overall process efficiency $(\mathrm{PE})$, which takes into account ME and $\mathrm{RE}$, 
was determined as the ratio of peak areas from pre-extraction spiked plasmas and post-extraction spiked water $(\mathrm{PE}=\mathrm{C} / \mathrm{A})$. For the post-extraction spiked water $(\mathrm{A})$, the mean peak area of the 3 determinations was used as reference for the calculations. For the 6 different plasmas (B and $\mathrm{C}$ ), the extractions were done in duplicate and the mean of each duplicate was considered. Variability between the different plasma batches was expressed as relative standard deviation (RSD). Normalized ME, RE and PE were calculated by dividing analytes' results by those of the respective IS [7].

\subsubsection{Linearity:}

Linearity was evaluated by using bulk spiked calibration curve standards and quality control standards. The calibration curve was constructed by using 9 non-zero calibration curve standard points spanning the range of $0.1-250.0 \mathrm{ng} / \mathrm{ml},(0.1,0.25,0.5,2.0,10.0,50.0,150.0,250.0 \mathrm{ng} / \mathrm{ml})$, four non-zero quality control standards $(0.1,0.3,75.6$ and $181.5 \mathrm{ng} / \mathrm{ml})$, and, in addition, a blank sample (spiked only with blank plasma), blank + IS sample (spiked only with blank plasma and IS sample). The chromatograms were shown for blank and LLOQ in Figure 2. Calibration curves were obtained by weighted $1 / \mathrm{x} 2$ linear regression model $(\mathrm{y}=\mathrm{mx}+\mathrm{c})$. The ratio of Almotriptan peak area to Naratriptan peak area was plotted against the concentration of Almotriptan in $\mathrm{ng} / \mathrm{ml}$. The suitability of the calibration curve was confirmed by back-calculating the concentrations of the calibration standards.

\subsubsection{Accuracy and Precision:}

Three precision and accuracy batches were performed in two days. Concentration values were obtained by the ratio between analytes' peak areas and those of the corresponding IS, recalculated with the daily calibration curve. Inter and intraday accuracy and precision values were expressed as \% CV. Accuracy should be within $85-115 \%$ of the theoretical value (LLOQ $80-120 \%$ ). Precision should be within $\pm 15 \%$ (LLOQ $\pm 20 \%$ ) Table 2 .

\subsubsection{Stability:}

Stability tests were performed at low $(0.3 \mathrm{ng} / \mathrm{ml})$ and high $(181.5 \mathrm{ng} / \mathrm{ml})$ control plasma samples. Following parameters were accessed, three freeze-and-thaw ( -20 to $25{ }^{\circ} \mathrm{C}$ ) cycles, Autosampler stability was performed at 24 and $48 \mathrm{hrs}$, Reinjection stability was performed for $24 \mathrm{hrs}$ and Bench top stability was performed at 4 and $6 \mathrm{hrs}$ as shown in Table 3. Subsequently, the Almotriptan concentrations were measured compared to freshly prepared samples. Stock solution stability was also done for 10 days at $2-8^{0} \mathrm{c}$.

\section{Results And Discussion:}

Development and validation of almotriptan in human plasma was done using HLB catridges followed by UPLC-MSMS analysis.All the validation results were meeting the acceptance criteria as new USFDA guidelines.

\subsection{Method Development and Optimization:}

The development of sensitive and selective bioanalytical method requires the judicious selection of chromatography column, mobile phase $\mathrm{pH}$ and organic solvent [8]. These parameters should be carefully monitored to produce the required resolution from endogenous components which in turn affect sensitivity and reproducibility of the analytical method by ion suppression. Once Chromatographic column, mobile phase $\mathrm{pH}$ and organic solvent are set then Gradient slope, flow rate, column temperature and buffer type and concentration can be manipulated for optimal response [9].The development of UPLC/MS/MS requires three separate steps like sample processing, LC method and MS method [10,11]. For sample processing $2 \times 4$ approach was tried with Oasis HLB, MCX, MAX, WCX cartridges, among which HLB cartridges gave good recovery and cleaner extracts with minimal matrix effect [12]. Then for UPLC [13] optimization Waters Aquity BEH C18, HSST3, CSH C18, were tried, HSST3 column gave good separation from endogenous components and sensitivity. Different $\mathrm{pH}$ acidic $\mathrm{pH} 20.1 \%$ formic acid, $5 \mathrm{mM}$ ammonium formate and $10 \mathrm{mM}$ Ammonium bicarbonate $\mathrm{pH} 9$ were tried in acidic and basic $\mathrm{pH}$ Almotriptan was eluting at the start of gradient and at $\mathrm{pH}$ of 6.2 analyte retained on the column and separated from endogenous components with good sensitivity $[14,15,16]$. Different buffers like ammonium acetate and ammonium formate were tried but ammonium acetate showed ion suppression so ammonium formate was used as buffer. Acetonitrile and methanol both were tried Acetonitrile gave good sensitivity. Once Column, mobile phase $\mathrm{pH}$ and organic solvent were set gradient slope and flow rate were optimized for good sensitivity and resolution [17]. Initial conditions were optimized from $5 \% \mathrm{~B}$ to $30 \% \mathrm{~B}$ in which $10 \% \mathrm{~B}$ found to be optimum. So the gradient slope was set from $10 \% \mathrm{~B}-90 \% \mathrm{~B}$. Different column temperatures were tried 30,40 , and $50^{\circ} \mathrm{c}$ not much variation was seen so column temperature was set to $40^{\circ} \mathrm{c}$. 


\subsection{Method Validation:}

Found this method to be linear from $0.1 \mathrm{ng} / \mathrm{ml}-250 \mathrm{ng} / \mathrm{ml}$. No interference was observed at the Retention time of analyte and IS in 6 processed blank plasma samples. Those results are in accordance with the limit of at least 5 recommended by the FDA. No carry-over was observed when blank plasma was analyzed just after the highest calibration standard. The relative standard deviation (R.S.D.) value for the interday and Intraday Precision and accuracy batches were found to be less than $10.0 \%$. Recovery was found to be between 80 to $85 \%$ for Low $(0.3 \mathrm{ng} / \mathrm{ml})$, medium $(75.6 \mathrm{ng} / \mathrm{ml})$, high $(181.5 \mathrm{ng} / \mathrm{ml})$ and $77 \%$ for Naratriptan. Bench top stability and Autosampler stability were found to be $6 \mathrm{hrs}$ and $48 \mathrm{hrs}$ respectively. Reinjection stability found to be $24 \mathrm{hrs}$. Stock solutions of Almotriptan and Naratriptan found to be stable for 13 days at $2-8^{0} \mathrm{c}$. The percentage stability of Almotriptan and Naratriptan was $102.44 \%$ and $103.37 \%$, respectively.

\section{Figures And Tables}<smiles>CN(C)CCc1c[nH]c2ccc(CS(=O)(=O)N3CCCC3)cc12</smiles>

(A)<smiles>CNS(=O)(=O)CCc1ccc2[nH]cc(C3CCN(C)CC3)c2c1</smiles>

(B)

Figure 1: Chemical structures of (A) Almotriptan and (B) Naratriptan.
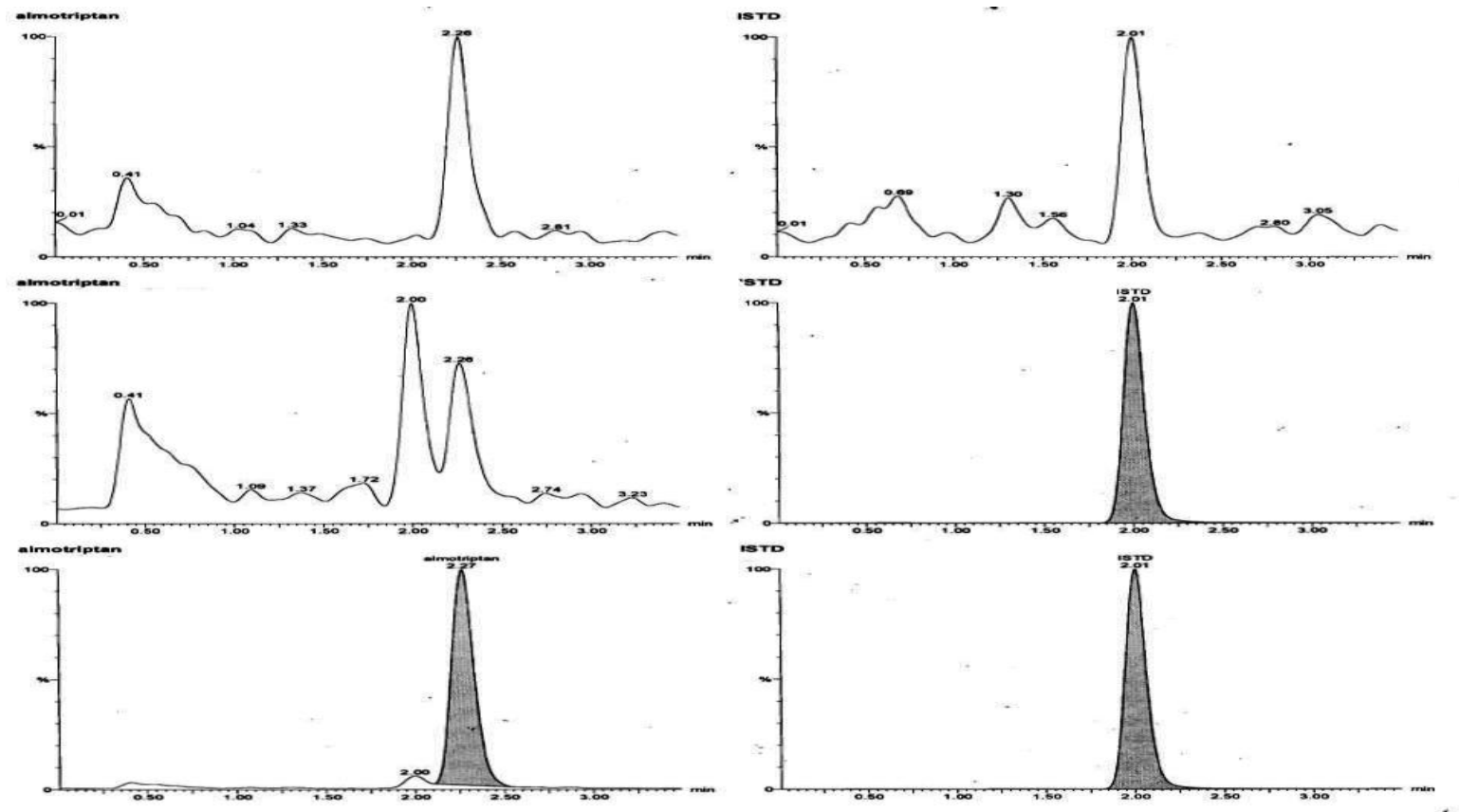

Figure 2: Typical MRM chromatograms of Blank, blank+IS and LLOQ for Almotriptan (left) and Naratriptan (right) 
Table 1: MRM parameters and voltages for Almotriptan and Naratriptan

\begin{tabular}{|c|c|c|c|c|}
\hline \multirow{2}{*}{ Almotriptan } & \multicolumn{4}{|c|}{ Nominal concentrations of quality control samples (ng/ml) } \\
\hline & 0.102 & 0.318 & 75.621 & 181.490 \\
\hline \multicolumn{5}{|l|}{ Intra day batch $(n=6)$} \\
\hline Accuracy $\%$ & $102.6 \%$ & $101.4 \%$ & $102.2 \%$ & $101.9 \%$ \\
\hline Precision $\%$ & $3.0 \%$ & $3.0 \%$ & $1.1 \%$ & $1.2 \%$ \\
\hline \multicolumn{5}{|l|}{ Inter day batch $(n=6)$} \\
\hline Accuracy $\%$ & $101.6 \%$ & $101.4 \%$ & $98.1 \%$ & $99.7 \%$ \\
\hline Precision $\%$ & $3.3 \%$ & $2.0 \%$ & $4.1 \%$ & $2.2 \%$ \\
\hline
\end{tabular}

Table 2: Precision and accuracy of the method for Almotriptan in human plasma.

\begin{tabular}{|lccc|}
\hline Compounds & MRM transition & cone voltage & collision energies \\
\hline Almotriptan & $336.26>157.04$ & 20 & 15 \\
Noratriptan & $336.26>98.11$ & 18 & 17 \\
& & & \\
\hline
\end{tabular}

Table 3: Stability results of Almotriptan in human plasma.

\begin{tabular}{|lcccc|}
\hline Stability Test & Almotriptan & & & \\
& Concentration & & & \\
& $(\mathrm{ng} / \mathrm{ml})$ & Mean \pm SD (ng/ml) & Accuracy $(\%)$ & Precision $(\%)$ \\
\hline Auto Sampler Stability & 0.318 & $0.308 \pm 0.01$ & 98.1 & $2.2 \%$ \\
(At $10^{0}$ c for 48 hrs) & 181.490 & $179.55 \pm 6.25$ & 99.4 & $1.1 \%$ \\
Freeze thaw stability (3) & 0.318 & $0.327 \pm 0.04$ & 99.6 & $3.0 \%$ \\
(At $10^{0}$ c for 48 hrs) & 181.490 & $174.22 \pm 3.25$ & 96.0 & $3.1 \%$ \\
Bench top stability (6hrs) & 0.318 & $0.320 \pm 0.03$ & 95.1 & $4.2 \%$ \\
& 181.490 & $185.35 \pm 7.26$ & 102.4 & $2.3 \%$ \\
Long term Stability & 0.318 & $0.292 \pm 0.01$ & 92.3 & $8.1 \%$ \\
(At -20 $0^{0}$ c for 30 days) & 181.490 & $180.66 \pm 1.56$ & 99.0 & $1.1 \%$ \\
Reinjection Stability ( 24hrs) & 0.318 & $0.322 \pm 0.03$ & 101.1 & $3.2 \%$ \\
& 181.490 & $182.85 \pm 3.1$ & 101.8 & $2.5 \%$ \\
\end{tabular}

\section{Conclusions:}

The combination of Solid phase extraction along with UPLC and XevoTQMS enables us to develop a simple fast and sensitive method for the determination of Almotriptan in human plasma. With sub $2 \mu$ particle size of the column helps to develop methods faster with good efficiency and the novel Z-spray design prevents unwanted and neutral compounds from entering analyzer of the instrument. Use of T-Wave optics and collision cell design allowed more than 12 points to be acquired along the very narrow peaks of UPLC. As per our knowledge this is the most sensitive method for determination of Almotriptan in human plasma, $0.1 \mathrm{ng} / \mathrm{ml}$ as LLOQ and with only $100 \mu$ l Of human plasma.This method is validated as per guide lines of US FDA so this method can be applied to pharmacokinetic studies.

\section{Conflict of interest}

The authors declare no conflict of interest.

\section{Acknowledgements}

I would like to thank my colleagues Mr.Kuttappa and Mr.James Nadar for laboratory support Dr.Gopal Vaidyanathan for practical assistance and Mrs. Neha Gudlawar for bibilical support.

\section{References:}

[1]. Almotriptan facts and comparisons. Drugs.com. Retrieved 7 October 2012

[2]. Kean, SJ; Goa, KL; Figgit, DP (2002)." Almotriptan its use in migraine.” Drugs 62 (2): 387-414

[3]. McEnroe, JD; Fleishaker, JC (2005). "Clinical pharmacokinetics of Almotriptan, a Serotonin 5-HT (1B/1D) receptor agonist for the treatment of migraine." Clinical pharmacokinetics 44 (3): 237-46.

[4]. Method Development and Validation of Almotriptan in Human Plasma by HPLC Tandem Mass Spectrometry: Application to Pharmacokinetic Study Sci Pharm. 2012; 80: 367-378 doi:10.3797/scipharm.1112-01. 
[5]. Food and Drug Administration, Guidance for Industry: Bioanalytical Method Validation 2001, http://www.fda.gov/downloads/Drugs/Guidance ComplianceRegulatoryInformation/Guidances/UCM070107.pdf (last access July 2012).

[6]. B.K. Matuszewski, M.L. Constanzer, C.M. Chavez-Eng, Strategies for the assessment of matrix effect in quantitative Bioanalytical methods based on HPLC-MS/MS, Anal. Chem. 75 (2003) 3019-3030.

[7]. Buprenorphine and norbuprenorphine quantification in human plasma by simple protein precipitation and ultra-high performance liquid chromatography tandem mass spectrometry.Journal of pharmaceutical and biomedical analysis 77 (2013) 1-8.

[8]. Electrospray and MALDI Mass Spectrometry Fundamentals, Instrumentation,Practicalities, and Biological Applications Second Edition Edited by Richard B. Cole.

[9]. A guide to effective method development in bioanalysis : Waters application note.

[10]. A systemic approach to Bioanalysis to LC method development using UPLC.Waters Application Note.

[11]. Novel Achievement of HPLC: UPLC. International Journal of PharmTech Research. Vol.3, No.3, pp1423-1429, July-Sept 2011.

[12]. Purity by SPE: Cleaner, simpler and faster SPE-LC/MS analysis. Waters oasis product application note.

[13]. Novel Achievement of HPLC: UPLC. International Journal of PharmTech Research. Vol.3, No.3, pp1423-1429, July-Sept 2011.

[14]. A review of current trends and advances in modern bio-analytical methods:Chromatography and sample preparation. Analytica Chimica Acta 656 (2009) 8-35.

[15]. R. Bonfiglio, R.C. King, T.V. Olah, K. Merkle, The effects of sample preparation methods on the variability of the electrospray ionization response for model drug compounds, Rapid Commun. Mass Spectrom. 13 (1999) 1175-1185.

[16]. A review of current trends and advances in modern bio-analytical methods:Chromatography and sample preparation. Analytica Chimica Acta 656 (2009) 8-35.

[17]. Effect of eluent on the ionization process in liquid chromatography-mass spectrometry Journal of Chromatography A, 1216 (2009) 685-699. 